\title{
An inverted $U$ relation existed between unemployment and foster home placements
}

\begin{abstract}
Catalano RA, Lind SL, Rosenblatt AB, et al. Unemployment and foster home placements: estimating the net effect of provocation and
\end{abstract} inhibition. Am J Public Health 1999 Jun;89:851-5.

\section{Question}

What is the association between unemployment and the prevalence of foster home placements?

\section{Design}

137 month population based cohort study.

\section{Setting}

California, USA.

\section{Participants}

People who resided in California during a 137 month period beginning in February 1984.

\section{Assessment of risk factors}

Increases in the seasonally adjusted unemployment rate for California were derived from estimates provided by the California Department of Employment Security. The rate was computed as the number of unemployed people divided by the sum of unemployed and employed people.

\section{Main outcome measures}

Monthly differences in the prevalence of foster home placements derived from data provided by the California Department of Social Services.

\begin{abstract}
Main results
The monthly prevalence of foster home placements in California ranged from 21 512-53 385 (mean 39 502). Monthly differences in the prevalence ranged from -2706 to 1745 (mean 234). The monthly unemployment rate ranged from $4.7-10.3 \%$ (mean 7.2\%). The mean seasonally adjusted change in the monthly unemployment rate was 0.012 . The statistical relation between monthly increases in the unemployment rate and changes in the prevalence of foster home placements was that of an inverted U. Thus, rising joblessness was associated with an increase in the prevalence of foster home placement. At a seasonally adjusted unemployment rate of approximately 0.15 , however, the net yield of foster placements for each unit of increase in the unemployment rate began to decrease indicating that the prevalence of foster placements increased when unemployment exceeded seasonal expectations (the effect of "provocation") but decreased when unemployment levels reached levels that frightened employed people into acting in a more socially acceptable manner to preserve their jobs (the "inhibition" effect)
\end{abstract}

\section{Conclusion}

The statistical relation between monthly increases in the unemployment rate and changes in the prevalence of foster home placements was that of an inverted U.

Source of funding: National Institute for Mental Health.

For correspondence: Dr R A Catalano, School of Public Health, University of California, Berkeley, CA 94720, USA.Fax +1 5106436981 .

\section{Commentary}

This study by Catalano et al found an inverted "U" shaped curve described the relation between seasonally adjusted increases in employment rates and foster care placements (a proxy measure for child abuse and neglect). The authors interpreted this as suggesting that when unemployment initially rises in a population, so do negative sequelae (the "provocation effect") but when unemployment continues to rise, people are more likely to control their behaviour when the penalty of job loss is perceived to be more certain (the "inhibition effect").

The most important limitation of this study is that we cannot be sure that it is children from homes where wage earners have been laid off who are admitted shortly afterwards to foster placements (the time lag entered into the statistical model is 1 month). Direct evidence at individual and macro-economic levels exists to link depression, violence, and alcohol abuse to unemployment, which allows the social and psychological mechanisms to be formulated. The findings, however, lead to testable hypotheses for short and medium term consequences of unemployment on abusive and neglectful behaviour that could be the focus of study.

An alternative explanation for the inhibition effect could be that the potential pool of foster placements available may be restricted. Once certain levels of occupancy of foster placement are reached, alternative social care interventions that result in the child staying at home or in another setting (eg, with relatives) may become increasingly used.
The notion of an inhibition effect may also offer clues to the development of effective strategies for intervention programmes. Aspects of individual and family coping mechanisms and decision making under stress, aside from increased "fearfulness" of job loss, could be supported in people who suffer job loss at an earlier point in the economic cycle. Building on these findings, work at the family level is required to identify what these protective factors might be and whether they could be promoted to support families in a proactive intervention programme.

Tony Charman, MSc, $\mathrm{PhD}, \mathrm{C}$ Psychol
Institute of Child Health
University College
London, UK 